\title{
Erratum to: Toroidal embeddings of abstractly planar graphs are knotted or linked
}

\author{
Senja Barthel ${ }^{1}$ • Dorothy Buck ${ }^{1}$
}

\section{Erratum to: J Math Chem (2015) 53:1772-1790} DOI 10.1007/s10910-015-0519-1

Unfortunately, the grant funding information was missed in the original publication. It has been corrected with this erratum.

The second author was partially supported by EPSRC Grants EP/G039585/1 and EP/H031367/1, and the Leverhulme Trust Grant RP2013-K-017.

The online version of the original article can be found under doi:10.1007/s10910-015-0519-1.

Senja Barthel

s.barthe111@imperial.ac.uk

Dorothy Buck

d.buck@imperial.ac.uk

1 Imperial College London, London, UK 